\title{
An Overview of Stock Portfolio Returns and Return Premia in Japan: The Case of Size and Book-to-Market Portfolios
}

\author{
Chikashi Tsuji ${ }^{1}$ \\ ${ }^{1}$ Faculty of Economics, Chuo University, Tokyo, Japan \\ Correspondence: Chikashi Tsuji, Professor, Faculty of Economics, Chuo University, 742-1 Higashinakano, Hachioji-shi, \\ Tokyo 192-0393, Japan.
}

Received: May 13, 2020

Accepted: May 27, $2020 \quad$ Available online: June 3, 2020

doi:10.11114/ijsss.v8i4.4885

URL: http://dx.doi.org/10.11114/ijsss.v8i4.4885

\begin{abstract}
This article explores Japanese stock portfolio returns and return premia by focusing on size- and book-to-market (BM)-sorted portfolios over the period of 1990 to 2020. As a result of our investigations, we derive the following useful findings. (1) In general, the value and/or size effects are continuously seen in the Japanese stock market. However, (2) these effects much depend on the economic and business background: for the performance of size- and BM-sorted portfolios in Japan, the value effect is stronger in some sub-periods; while the size effect is clearer in other sub-periods. Furthermore, (3) this study employs the data in US dollars, and computes various statistics and measures for both our full sample period and many different sub-periods, whose economic circumstances are rather different. Therefore, not only for academic researchers but also for international investors, our findings shall be highly beneficial for enriching the understanding of Japanese stock portfolio returns and return premia.
\end{abstract}

Keywords: Japanese stock market, portfolio return, return premia, size effect, value effect

\section{Introduction}

Research of stock returns and return premia of size- and book-to-market (BM)-sorted portfolios is important because size and BM ratio are key elements for effective constructions of quantitative portfolios, for which higher returns are expected (see, for example, Fama and French, 1993; Daniel and Titman, 1997). In contrast, for the Japanese stock market, not many research from this viewpoint can be seen in existing studies; hence, conducting an overview of size and BM portfolios for Japanese stocks in a well-organized manner is highly meaningful. Based on this motivation, this article explores Japanese stock returns and return premia by focusing on size- and BM-sorted portfolios over the period of 1990 to 2020.

As a result of our examinations, we derive the following interesting and practically useful findings. (1) In general, the value and/or size effects are continuously seen in the Japanese stock market. However, (2) these effects greatly depend on the economic conditions: regarding the performance of size- and BM-sorted portfolios in Japan, the value effect is stronger in some sub-periods; while the size effect is clearer in other sub-periods. (3) We emphasize that this paper uses the data in US dollars, and computes various statistics and measures for both our full sample period and many different sub-periods, whose economic circumstances are rather different. Therefore, not only for Japanese investors but also for international investors, our above findings shall be highly beneficial for deepening the understanding of Japanese stock portfolio returns and return premia, which were not enough investigated in existing literature.

The rest of this article is as follows. First, Section 2 conducts a brief related literature review; and Section 3 documents our data and methodology for our investigations. Afterwards, Section 4 explains our main findings; and Section 5 presents our conclusions.

\section{Brief Literature Review}

This section conducts a brief related literature review. First, well-known paper by Fama and French (1993) evidenced the importance of the size and value effects in the US stock market, and they suggested their three-factor asset pricing model. Afterwards, in the US, Fama and French (1995) studied whether the stock price behavior, in relation to size and BM, reflects the earnings behavior. Later, Fama and French (1998) tested the performance of value stocks and growth stocks in international stock markets; and further, Fama and French (2012) examined the size, value, and momentum 
effects also in international stock markets.

As for Japan, Chan et al. (1991) examined the relations of cross-sectional return differences of Japanese stocks with the differences of the earnings yield, size, BM ratio, and cash flow yield. Later, after empirical examinations, Daniel et al. (2001) rejected the Fama and French (1993) three-factor model, but reported that in Japan, they failed to reject their characteristic model advocated by Daniel and Titman (1997). Further, in the size- and BM-sorted portfolios in Japan over the period from 1981 to 2005, Tsuji (2010) found the positive return premia unexplained by macroeconomic factors. Also, Tsuji (2012) analyzed Japanese size and BM portfolios from 1981 to 2005, and found the positive return premia unexplained by the Fama and French (1993) three-factor model. We note that Japanese stock portfolio analysis that covered until very recent periods is rather limited although some international asset pricing research for international stock markets, such studies as Fama and French (2012, 2017), included the Japanese cases as part of them.

As stressed above, there exist not many studies that focused on the size and value effects in Japan; thus we consider that our overview of the returns and return premia of Japanese size- and BM-sorted portfolios for about 30 years should provide precious information both for academic researchers and industry practitioners.

\section{Data and Methods}

This section first explains our data. All basic data of Japanese stock portfolios are percentage returns in US dollars and were supplied by Eugene Fama and Kenneth French. Specifically, Mkt denotes the market portfolio; Small-Low BM denotes the small-sized and low BM portfolio; and Small-Neutral is the small-sized and neutral BM portfolio. Further, Small-High BM denotes the small-sized and high BM portfolio; Big-Low BM is the big-sized and low BM portfolio; Big-Neutral is the big-sized and neutral BM portfolio; and Big-High BM denotes the big-sized and high BM portfolio. We employ simple statistical and graphical analyses in this study.

This paper first analyzes our full sample period and the first half and second half sub-periods, which are from July 1990 to March 2020, from July 1990 to December 2005, and from January 2006 to March 2020. Afterwards, we further analyze six five-year sub-periods of (1) July 1990 to June 1995, including the Japanese stock price bubble collapse period; (2) July 1997 to June 2002, including the IT bubble period; (3) January 2002 to December 2006, when Japan's Quantitative Easing (QE) boosted Japanese stock prices; (4) January 2007 to December 2011, including the US subprime crisis and Lehman collapse; (5) April 2010 to March 2015, including the Great East Japan Earthquake; and (6) April 2015 to March 2020, including successive monetary easing policies undertaken by the Bank of Japan (BOJ).

Figure 1 shows the time-series evolution of cumulative raw returns of the above six Japanese size and BM portfolios with cumulative raw returns of the market portfolio. Specifically, Panel A of this figure plots those for our full sample period, Panel B shows those for our first half period, and Panel C exhibits those for our second half period. In Panels A and B, all prices equal to 100 in July 1990, and in Panel C, all prices equal to 100 in January 2006. Moreover, Figure 2 plots the time-series evolution of cumulative return premia of the above six Japanese size and BM portfolios over the market portfolio. More concretely, Panel A of this figure plots those for our full sample period, Panel B shows those for our first half period, and Panel C presents those for our second half period. In Panels A and B of Figure 2, all premia equal to zero in July 1990, and in Panel C, all premia equal to zero in January 2006.

\section{Main Findings}

We compute and show several statistics for our full period and the first half and second half sub-periods in Table 1, and present those for our six five-year sub-periods in Table 2. Explaining our main findings, first, as in Panel A of Table 1, for our full period, Small-High BM portfolio returns are the highest and Big-High BM portfolio returns are the second highest. Second, as Panel B of Table 1 shows, for our first half period, Big-High BM portfolio returns are the highest and Small-High BM portfolio returns are the second highest. Further, as in Panel C of Table 1, for our second half period, Small-High BM portfolio returns are the highest and Small-Neutral BM portfolio returns are the second highest.

In addition, as in Panel A of Table 2, for the period including the Japanese stock price bubble collapse, Big-High BM portfolio returns are the highest and Small-High BM portfolio returns are the second highest. Second, as Panel B of Table 2 shows, for the period including the IT bubble, Big-High BM portfolio returns are the highest and Big-Neutral BM portfolio returns are the second highest. Third, as in Panel C of Table 2, for the period when Japan's QE boosted stock prices in Japan, Small-High BM portfolio returns are the highest and Small-Neutral BM portfolio returns are the second highest. Fourth, for the period including the US subprime crisis and Lehman collapse, as Panel D of Table 2 exhibits, Small-High BM portfolio returns are the highest and Small-Neutral BM portfolio returns are the second highest. Fifth, as in Panel E of Table 2, for the period including the Great East Japan Earthquake, Small-Low BM portfolio returns are the highest and Small-High BM portfolio returns are the second highest. Sixth, as in Panel F of Table 2, for the period of successive monetary easing policies by the BOJ, Small-Neutral BM portfolio returns are the highest and Small-Low BM portfolio returns are the second highest. 
Panel A. July 1990 to March 2020

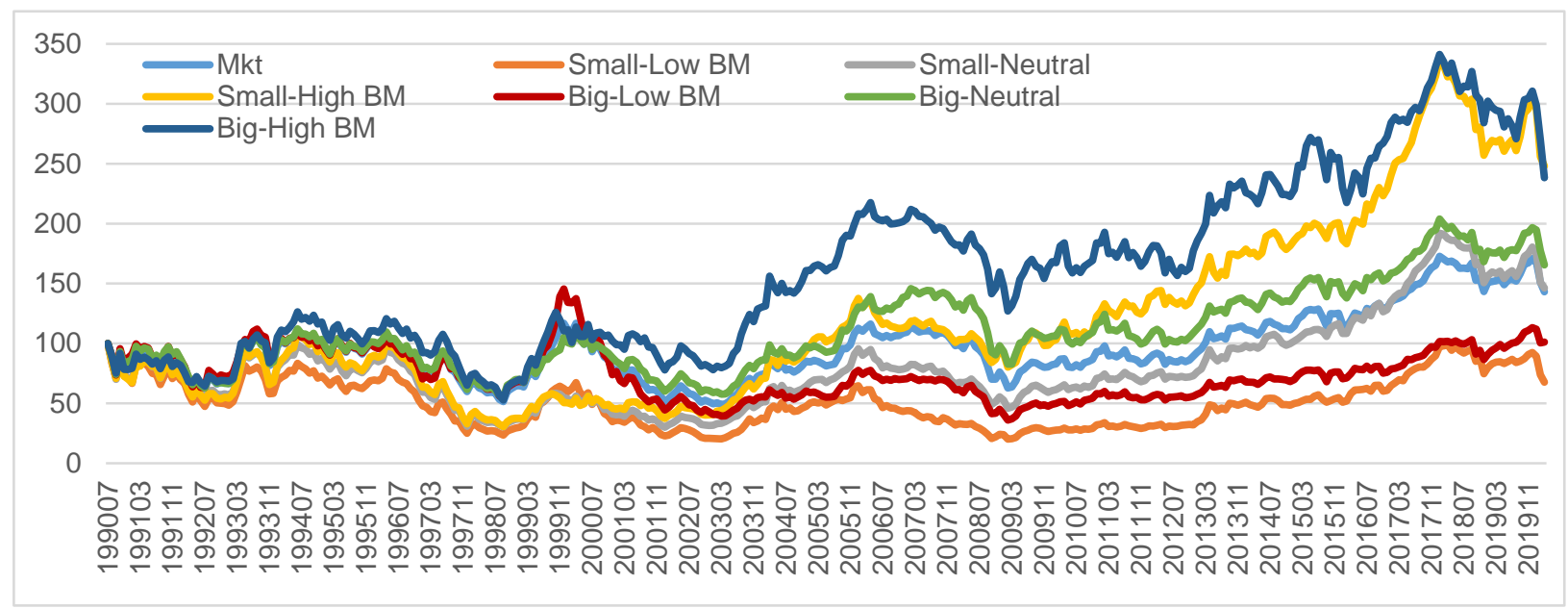

Panel B. July 1990 to December 2005

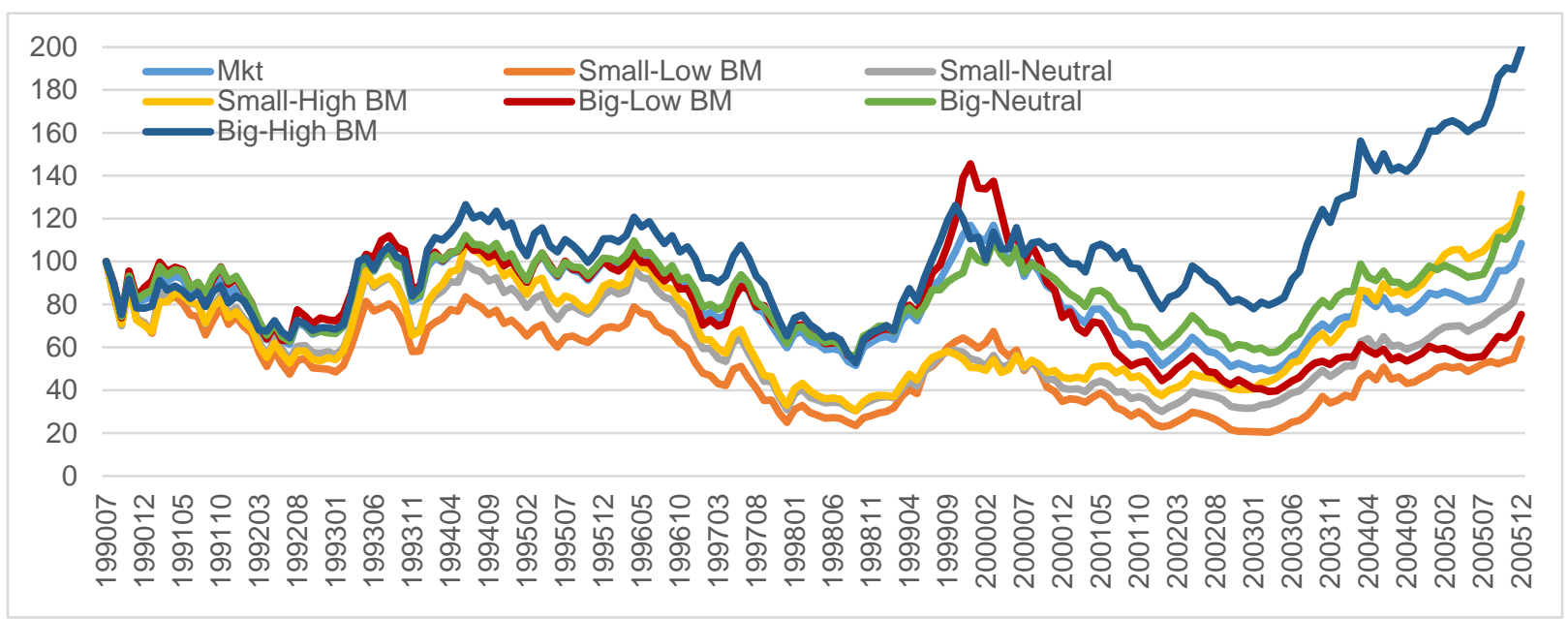

Panel C. January 2006 to March 2020

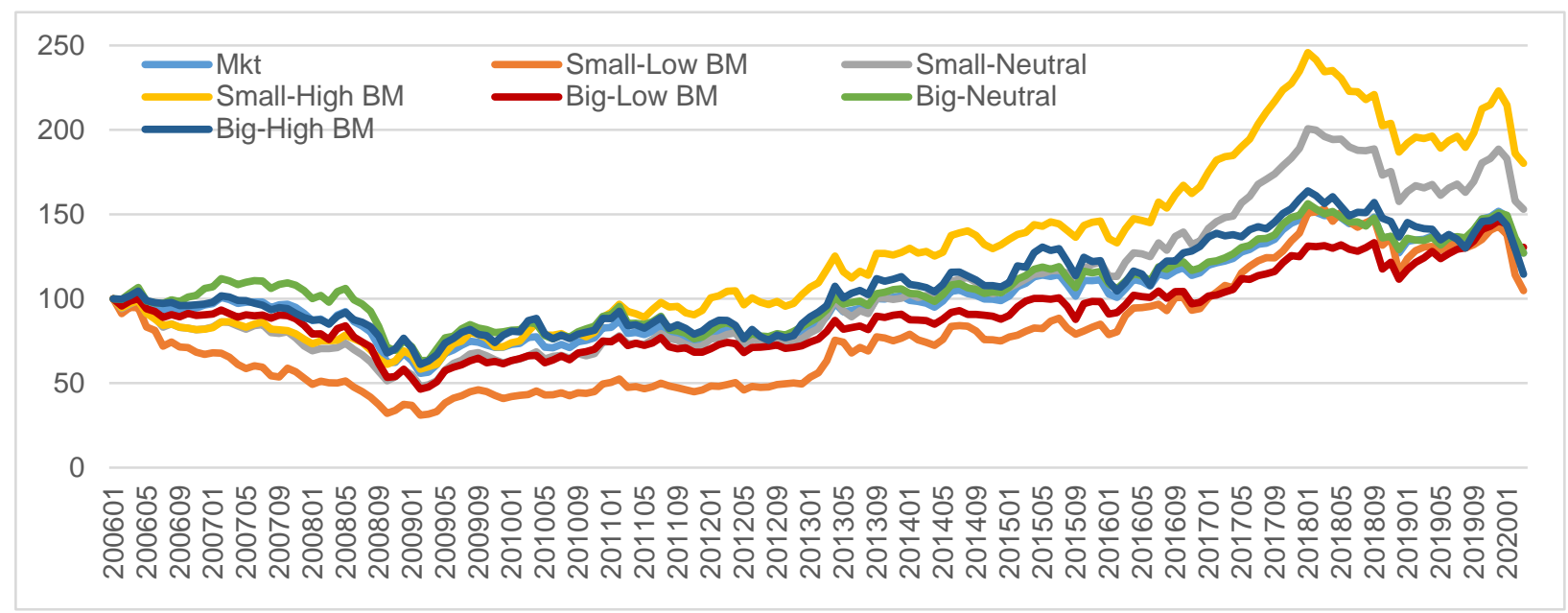

Figure 1. Evolution of cumulative raw returns of Japanese size- and BM-sorted portfolios 
Panel A. July 1990 to March 2020

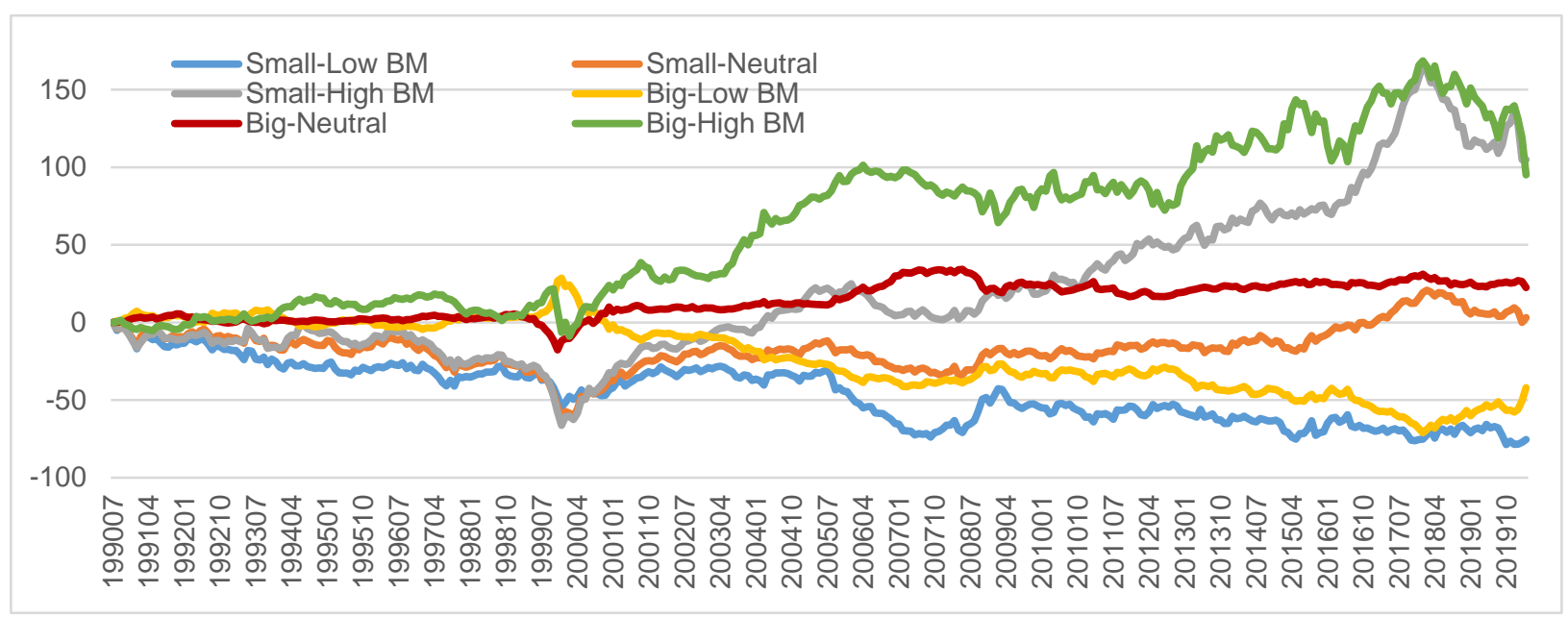

Panel B. July 1990 to December 2005

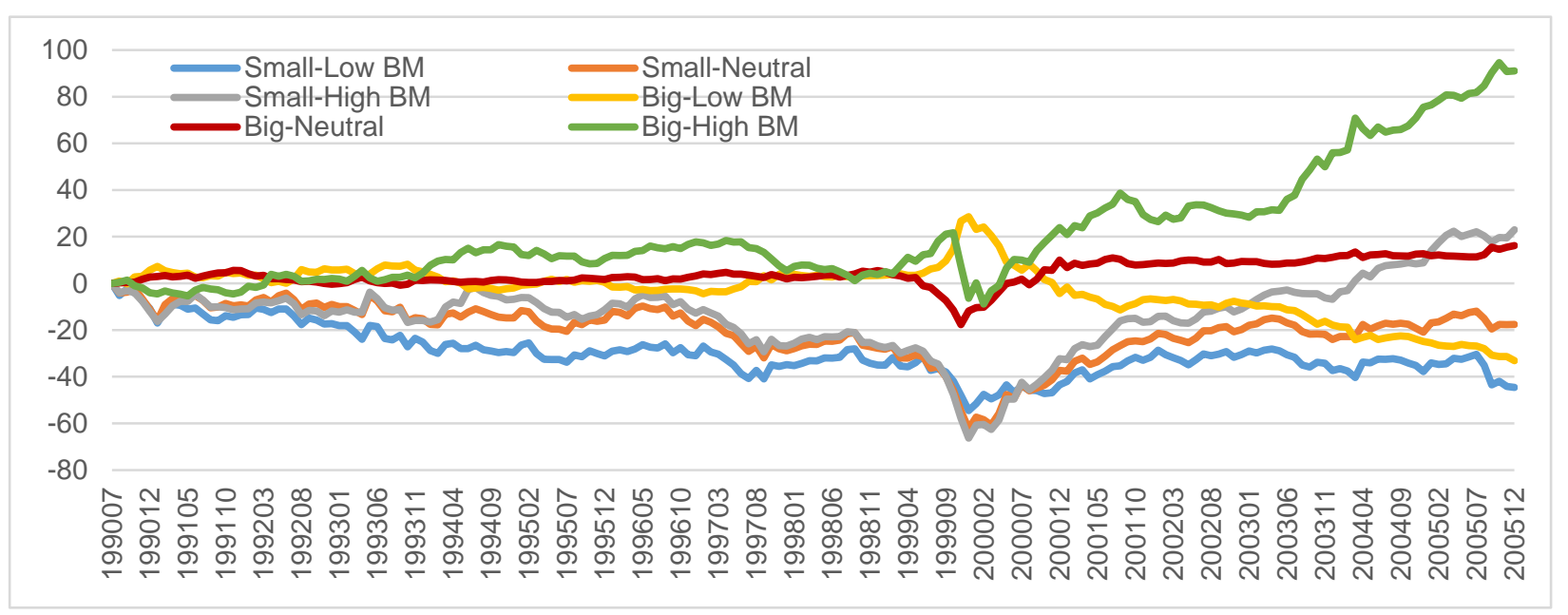

Panel C. January 2006 to March 2020

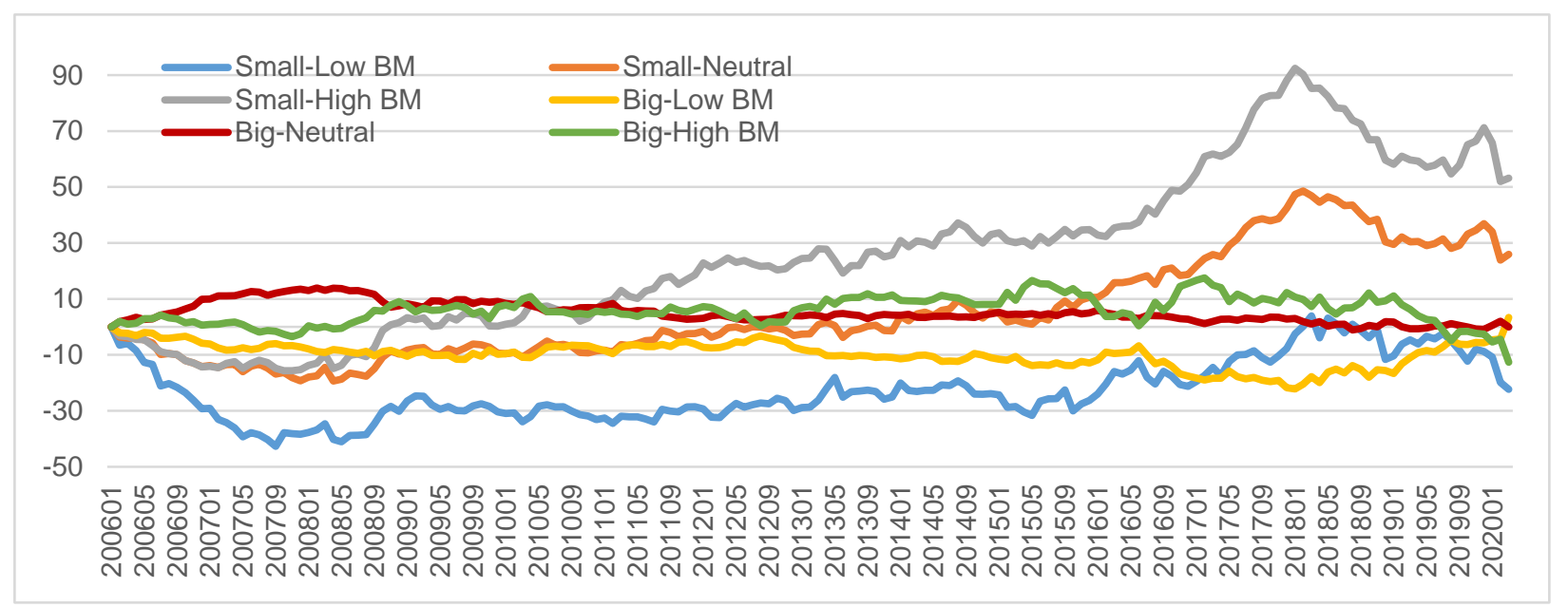

Figure 2. Evolution of cumulative return premia of Japanese size- and BM-sorted portfolios over the market portfolio 
Table 1. Statistics for Japanese size- and BM-sorted portfolio returns and return premia: Full and two sub-periods

Panel A. July 1990 to March 2020

\begin{tabular}{|c|c|c|c|c|c|c|c|}
\hline \multicolumn{8}{|c|}{ A-1. Portfolio percentage raw return } \\
\hline \multicolumn{8}{|c|}{ Monthly statistics } \\
\hline & Mkt & $\begin{array}{l}\text { Small- } \\
\text { Low BM }\end{array}$ & $\begin{array}{l}\text { Small- } \\
\text { Neutral }\end{array}$ & $\begin{array}{l}\text { Small- } \\
\text { High BM }\end{array}$ & $\begin{array}{l}\text { Big- } \\
\text { Low BM }\end{array}$ & $\begin{array}{l}\text { Big- } \\
\text { Neutral }\end{array}$ & $\begin{array}{l}\text { Big- } \\
\text { High BM }\end{array}$ \\
\hline Mean & 0.26 & 0.18 & 0.33 & 0.49 & 0.18 & 0.29 & 0.43 \\
\hline Maximum & 25.58 & 27.96 & 29.33 & 25.05 & 29.66 & 25.59 & 21.78 \\
\hline Minimum & -16.78 & -19.32 & -18.78 & -18.21 & -17.88 & -16.55 & -16.59 \\
\hline SD & 5.64 & 7.54 & 6.55 & 6.51 & 6.04 & 5.50 & 6.03 \\
\hline Skewness & 0.36 & 0.41 & 0.46 & 0.40 & 0.30 & 0.46 & 0.46 \\
\hline Kurtosis & 1.49 & 1.18 & 2.29 & 1.56 & 1.98 & 1.80 & 1.18 \\
\hline \multicolumn{8}{|c|}{ Annualized statistics } \\
\hline & Mkt & $\begin{array}{l}\text { Small- } \\
\text { Low BM }\end{array}$ & $\begin{array}{l}\text { Small- } \\
\text { Neutral }\end{array}$ & $\begin{array}{l}\text { Small- } \\
\text { High BM }\end{array}$ & $\begin{array}{l}\text { Big- } \\
\text { Low BM }\end{array}$ & $\begin{array}{l}\text { Big- } \\
\text { Neutral }\end{array}$ & $\begin{array}{l}\text { Big- } \\
\text { High BM }\end{array}$ \\
\hline Return & 3.12 & 2.18 & 3.99 & 5.84 & 2.17 & 3.49 & 5.11 \\
\hline SD & 19.54 & 26.13 & 22.67 & 22.55 & 20.93 & 19.06 & 20.90 \\
\hline Risk premia & 0.53 & -0.41 & 1.40 & 3.25 & -0.43 & 0.90 & 2.52 \\
\hline Sharp ratio & 0.03 & -0.02 & 0.06 & 0.14 & -0.02 & 0.05 & 0.12 \\
\hline
\end{tabular}

A-2. Portfolio percentage return premia over the market portfolio

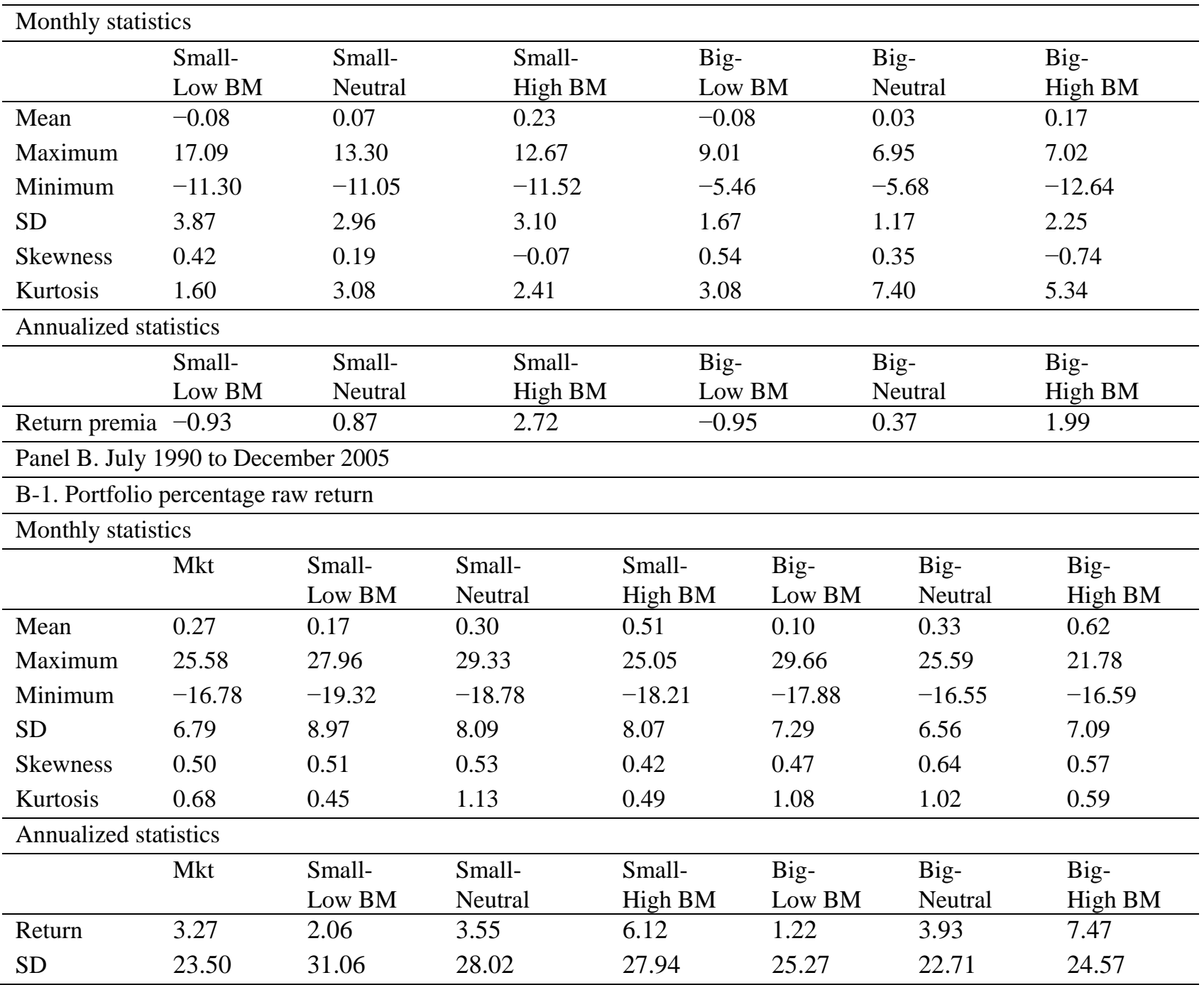




\begin{tabular}{|c|c|c|c|c|c|c|c|}
\hline Risk premia & -0.64 & -1.86 & -0.36 & 2.20 & -2.70 & 0.02 & 3.56 \\
\hline Sharp ratio & -0.03 & -0.06 & -0.01 & 0.08 & -0.11 & 0.00 & 0.14 \\
\hline \multicolumn{8}{|c|}{ B-2. Portfolio percentage return premia over the market portfolio } \\
\hline \multicolumn{8}{|c|}{ Monthly statistics } \\
\hline & $\begin{array}{l}\text { Small- } \\
\text { Low BM }\end{array}$ & $\begin{array}{l}\text { Small- } \\
\text { Neutral }\end{array}$ & \multicolumn{2}{|l|}{$\begin{array}{l}\text { Small- } \\
\text { High BM }\end{array}$} & $\begin{array}{l}\text { Big- } \\
\text { Low BM }\end{array}$ & $\begin{array}{l}\text { Big- } \\
\text { Neutral }\end{array}$ & $\begin{array}{l}\text { Big- } \\
\text { High BM }\end{array}$ \\
\hline Mean & -0.10 & 0.02 & \multicolumn{2}{|l|}{0.24} & -0.17 & 0.06 & 0.35 \\
\hline Maximum & 17.09 & 13.30 & \multicolumn{2}{|l|}{12.67} & 9.01 & 6.95 & 7.02 \\
\hline Minimum & -11.30 & -11.05 & \multicolumn{2}{|l|}{-11.52} & -5.46 & -5.68 & -12.64 \\
\hline SD & 4.32 & 3.67 & \multicolumn{2}{|l|}{3.79} & 1.88 & 1.43 & 2.58 \\
\hline Skewness & 0.61 & 0.24 & \multicolumn{2}{|l|}{-0.10} & 0.65 & 0.34 & -1.14 \\
\hline Kurtosis & 1.61 & 1.79 & 1.36 & & 3.24 & 5.75 & 5.74 \\
\hline \multicolumn{8}{|c|}{ Annualized statistics } \\
\hline & $\begin{array}{l}\text { Small- } \\
\text { Low BM }\end{array}$ & $\begin{array}{l}\text { Small- } \\
\text { Neutral }\end{array}$ & \multicolumn{2}{|l|}{$\begin{array}{l}\text { Small- } \\
\text { High BM }\end{array}$} & $\begin{array}{l}\text { Big- } \\
\text { Low BM }\end{array}$ & $\begin{array}{l}\text { Big- } \\
\text { Neutral }\end{array}$ & $\begin{array}{l}\text { Big- } \\
\text { High BM }\end{array}$ \\
\hline Return premia & -1.21 & 0.28 & 2.85 & & -2.05 & 0.66 & 4.20 \\
\hline Panel C. Janua & ry 2006 to 1 & $\operatorname{arch} 2020$ & & & & & \\
\hline C-1. Portfolio & percentage & $w$ return & & & & & \\
\hline Monthly statis & & & & & & & \\
\hline & Mkt & $\begin{array}{l}\text { Small- } \\
\text { Low BM }\end{array}$ & $\begin{array}{l}\text { Small- } \\
\text { Neutral }\end{array}$ & $\begin{array}{l}\text { Small- } \\
\text { High BI }\end{array}$ & $\begin{array}{l}\text { Big- } \\
\text { Low BM }\end{array}$ & $\begin{array}{l}\text { Big- } \\
\text { Neutral }\end{array}$ & $\begin{array}{l}\text { Big- } \\
\text { High BM }\end{array}$ \\
\hline Mean & 0.25 & 0.19 & 0.37 & 0.46 & 0.27 & 0.25 & 0.21 \\
\hline Maximum & 10.89 & 19.30 & 12.55 & 11.34 & 13.09 & 10.86 & 12.00 \\
\hline Minimum & -13.47 & -17.32 & -13.64 & -13.35 & -13.88 & -14.85 & -13.83 \\
\hline SD & 4.06 & 5.63 & 4.30 & 4.24 & 4.30 & 4.07 & 4.62 \\
\hline Skewness & -0.51 & -0.19 & -0.35 & -0.19 & -0.62 & -0.59 & -0.26 \\
\hline Kurtosis & 1.04 & 1.26 & 0.77 & 0.66 & 1.42 & 1.24 & 0.48 \\
\hline Annualized sta & tistics & & & & & & \\
\hline & Mkt & $\begin{array}{l}\text { Small- } \\
\text { Low BM }\end{array}$ & $\begin{array}{l}\text { Small- } \\
\text { Neutral }\end{array}$ & $\begin{array}{l}\text { Small- } \\
\text { High BI }\end{array}$ & $\begin{array}{l}\text { Big- } \\
\text { Low BM }\end{array}$ & $\begin{array}{l}\text { Big- } \\
\text { Neutral }\end{array}$ & $\begin{array}{l}\text { Big- } \\
\text { High BM }\end{array}$ \\
\hline Return & 2.95 & 2.32 & 4.47 & 5.54 & 3.20 & 3.00 & 2.53 \\
\hline SD & 14.08 & 19.50 & 14.91 & 14.67 & 14.90 & 14.11 & 16.01 \\
\hline Risk premia & 1.80 & 1.17 & 3.31 & 4.39 & 2.04 & 1.85 & 1.38 \\
\hline Sharp ratio & 0.13 & 0.06 & 0.22 & 0.30 & 0.14 & 0.13 & 0.09 \\
\hline C-2. Portfolio & percentage & urn premia c & r the market port & folio & & & \\
\hline Monthly statis & & & & & & & \\
\hline & $\begin{array}{l}\text { Small- } \\
\text { Low BM }\end{array}$ & $\begin{array}{l}\text { Small- } \\
\text { Neutral }\end{array}$ & $\begin{array}{l}\text { Small- } \\
\text { High BM }\end{array}$ & & $\begin{array}{l}\text { Big- } \\
\text { Low BM }\end{array}$ & $\begin{array}{l}\text { Big- } \\
\text { Neutral }\end{array}$ & $\begin{array}{l}\text { Big- } \\
\text { High BM }\end{array}$ \\
\hline Mean & -0.05 & 0.13 & 0.22 & & 0.02 & 0.00 & -0.03 \\
\hline Maximum & 10.35 & 5.16 & 8.10 & & 5.75 & 2.80 & 5.66 \\
\hline Minimum & -9.77 & -5.61 & -5.96 & & -3.53 & -2.69 & -6.47 \\
\hline SD & 3.32 & 1.92 & 2.12 & & 1.41 & 0.81 & 1.80 \\
\hline Skewness & -0.03 & -0.13 & 0.16 & & 0.40 & -0.01 & 0.29 \\
\hline Kurtosis & 0.60 & 0.11 & 0.91 & & 1.27 & 1.05 & 1.19 \\
\hline Annualized sta & tistics & & & & & & \\
\hline & $\begin{array}{l}\text { Small- } \\
\text { Low BM }\end{array}$ & $\begin{array}{l}\text { Small- } \\
\text { Neutral }\end{array}$ & $\begin{array}{l}\text { Small- } \\
\text { High BM }\end{array}$ & & $\begin{array}{l}\text { Big- } \\
\text { Low BM }\end{array}$ & $\begin{array}{l}\text { Big- } \\
\text { Neutral }\end{array}$ & $\begin{array}{l}\text { Big- } \\
\text { High BM }\end{array}$ \\
\hline Return premia & -0.63 & 1.52 & 2.59 & & 0.25 & 0.05 & -0.41 \\
\hline
\end{tabular}

Notes: Monthly observation numbers: 357 (Panel A), 186 (Panel B), and 171 (Panel C). SD: standard deviation. 
Table 2. Statistics for Japanese size- and BM-sorted portfolio returns and return premia: Six five-year sub-periods

Panel A. July 1990 to June 1995

\begin{tabular}{|c|c|c|c|c|c|c|c|}
\hline \multicolumn{8}{|c|}{ A-1. Portfolio percentage raw return } \\
\hline \multicolumn{8}{|c|}{ Monthly statistics } \\
\hline & Mkt & $\begin{array}{l}\text { Small- } \\
\text { Low BM }\end{array}$ & $\begin{array}{l}\text { Small- } \\
\text { Neutral }\end{array}$ & $\begin{array}{l}\text { Small- } \\
\text { High BM }\end{array}$ & $\begin{array}{l}\text { Big- } \\
\text { Low BM }\end{array}$ & $\begin{array}{l}\text { Big- } \\
\text { Neutral }\end{array}$ & $\begin{array}{l}\text { Big- } \\
\text { High BM }\end{array}$ \\
\hline Mean & 0.22 & -0.31 & 0.04 & 0.23 & 0.22 & 0.22 & 0.43 \\
\hline Maximum & 25.58 & 26.27 & 29.33 & 25.05 & 29.66 & 25.59 & 21.78 \\
\hline Minimum & -16.78 & -19.32 & -18.78 & -18.21 & -17.88 & -16.55 & -16.59 \\
\hline SD & 8.30 & 9.94 & 9.92 & 9.78 & 8.62 & 8.28 & 8.31 \\
\hline Skewness & 0.61 & 0.60 & 0.69 & 0.48 & 0.74 & 0.71 & 0.56 \\
\hline Kurtosis & 0.84 & 0.27 & 0.80 & 0.03 & 1.78 & 0.84 & 0.61 \\
\hline \multicolumn{8}{|c|}{ Annualized statistics } \\
\hline & Mkt & $\begin{array}{l}\text { Small- } \\
\text { Low BM }\end{array}$ & $\begin{array}{l}\text { Small- } \\
\text { Neutral }\end{array}$ & $\begin{array}{l}\text { Small- } \\
\text { High BM }\end{array}$ & $\begin{array}{l}\text { Big- } \\
\text { Low BM }\end{array}$ & $\begin{array}{l}\text { Big- } \\
\text { Neutral }\end{array}$ & $\begin{array}{l}\text { Big- } \\
\text { High BM }\end{array}$ \\
\hline Return & 2.59 & -3.70 & 0.43 & 2.80 & 2.65 & 2.68 & 5.11 \\
\hline SD & 28.75 & 34.42 & 34.38 & 33.89 & 29.86 & 28.67 & 28.80 \\
\hline Risk premia & -1.84 & -8.13 & -4.00 & -1.63 & -1.78 & -1.75 & 0.69 \\
\hline Sharp ratio & -0.06 & -0.24 & -0.12 & -0.05 & -0.06 & -0.06 & 0.02 \\
\hline
\end{tabular}

A-2. Portfolio percentage return premia over the market portfolio

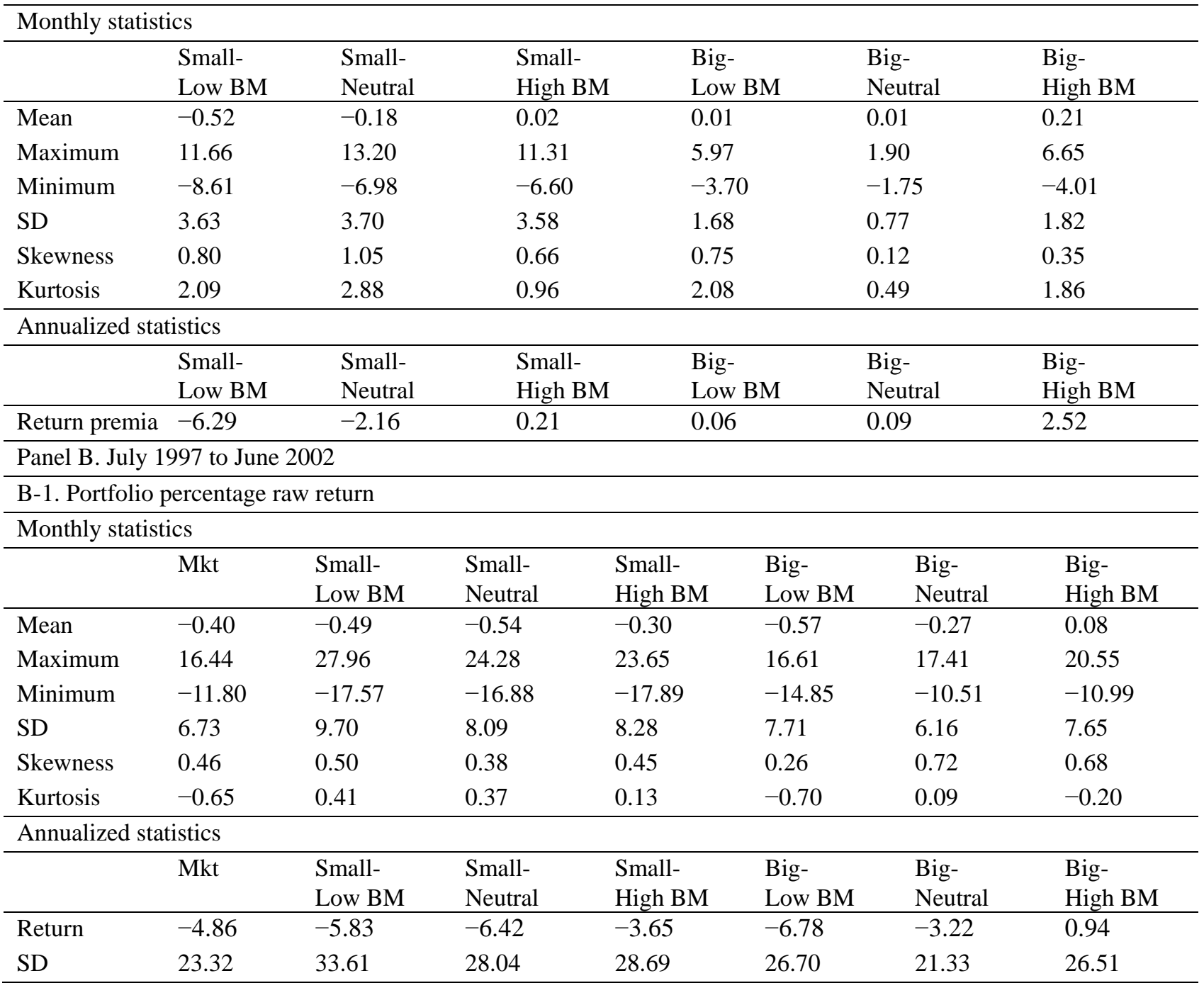




\begin{tabular}{|c|c|c|c|c|c|c|c|}
\hline Risk premia & -9.30 & -10.27 & -10.87 & -8.09 & -11.22 & -7.66 & -3.50 \\
\hline Sharp ratio & -0.40 & -0.31 & -0.39 & -0.28 & -0.42 & -0.36 & -0.13 \\
\hline \multicolumn{8}{|c|}{ B-2. Portfolio percentage return premia over the market portfolio } \\
\hline \multicolumn{8}{|c|}{ Monthly statistics } \\
\hline & $\begin{array}{l}\text { Small- } \\
\text { Low BM }\end{array}$ & $\begin{array}{l}\text { Small- } \\
\text { Neutral }\end{array}$ & \multicolumn{2}{|l|}{$\begin{array}{l}\text { Small- } \\
\text { High BM } \\
\end{array}$} & $\begin{array}{l}\text { Big- } \\
\text { Low BM }\end{array}$ & $\begin{array}{l}\text { Big- } \\
\text { Neutral }\end{array}$ & $\begin{array}{l}\text { Big- } \\
\text { High BM }\end{array}$ \\
\hline Mean & -0.08 & -0.13 & \multicolumn{2}{|l|}{0.10} & -0.16 & 0.14 & 0.48 \\
\hline Maximum & 17.09 & 13.30 & \multicolumn{2}{|l|}{12.67} & 9.01 & 6.95 & 7.02 \\
\hline Minimum & -11.30 & -11.05 & \multicolumn{2}{|l|}{-11.52} & -5.46 & -5.68 & -12.64 \\
\hline SD & 5.11 & 4.39 & \multicolumn{2}{|l|}{4.89} & 2.48 & 2.26 & 3.85 \\
\hline Skewness & 0.64 & -0.11 & \multicolumn{2}{|l|}{-0.32} & 0.64 & 0.15 & -1.17 \\
\hline Kurtosis & 1.81 & 1.29 & \multicolumn{2}{|l|}{0.69} & 2.13 & 1.44 & 2.57 \\
\hline \multicolumn{8}{|c|}{ Annualized statistics } \\
\hline & $\begin{array}{l}\text { Small- } \\
\text { Low BM }\end{array}$ & $\begin{array}{l}\text { Small- } \\
\text { Neutral } \\
\end{array}$ & \multicolumn{2}{|l|}{$\begin{array}{l}\text { Small- } \\
\text { High BM } \\
\end{array}$} & $\begin{array}{l}\text { Big- } \\
\text { Low BM }\end{array}$ & $\begin{array}{l}\text { Big- } \\
\text { Neutral }\end{array}$ & $\begin{array}{l}\text { Big- } \\
\text { High BM }\end{array}$ \\
\hline Return premia & -0.97 & -1.57 & 1.21 & & -1.92 & 1.64 & 5.80 \\
\hline Panel C. Januar & ry 2002 to 1 & cember 2006 & & & & & \\
\hline C-1. Portfolio $\mathrm{p}$ & percentage & return & & & & & \\
\hline Monthly statisti & & & & & & & \\
\hline & Mkt & $\begin{array}{l}\text { Small- } \\
\text { Low BM }\end{array}$ & $\begin{array}{l}\text { Small- } \\
\text { Neutral } \\
\end{array}$ & $\begin{array}{l}\text { Small- } \\
\text { High BM }\end{array}$ & $\begin{array}{l}\text { Big- } \\
\text { Low BM }\end{array}$ & $\begin{array}{l}\text { Big- } \\
\text { Neutral }\end{array}$ & $\begin{array}{l}\text { Big- } \\
\text { High BM }\end{array}$ \\
\hline Mean & 1.22 & 1.23 & 1.65 & 1.90 & 0.71 & 1.40 & 1.60 \\
\hline Maximum & 15.12 & 23.00 & 21.85 & 21.79 & 11.69 & 14.79 & 18.95 \\
\hline Minimum & -7.68 & -12.13 & -8.91 & -7.41 & -9.47 & -8.33 & -6.32 \\
\hline SD & 4.74 & 7.16 & 5.40 & 5.26 & 4.90 & 4.68 & 4.90 \\
\hline Skewness & 0.31 & 0.50 & 0.62 & 0.81 & 0.05 & 0.21 & 0.96 \\
\hline Kurtosis & 0.04 & 0.64 & 2.19 & 2.17 & -0.58 & 0.12 & 1.68 \\
\hline Annualized stat & tistics & & & & & & \\
\hline & Mkt & $\begin{array}{l}\text { Small- } \\
\text { Low BM }\end{array}$ & $\begin{array}{l}\text { Small- } \\
\text { Neutral }\end{array}$ & $\begin{array}{l}\text { Small- } \\
\text { High BM }\end{array}$ & $\begin{array}{l}\text { Big- } \\
\text { Low BM }\end{array}$ & $\begin{array}{l}\text { Big- } \\
\text { Neutral }\end{array}$ & $\begin{array}{l}\text { Big- } \\
\text { High BM }\end{array}$ \\
\hline Return & 14.68 & 14.75 & 19.80 & 22.79 & 8.55 & 16.74 & 19.20 \\
\hline SD & 16.43 & 24.80 & 18.71 & 18.20 & 16.98 & 16.21 & 16.97 \\
\hline Risk premia & 12.39 & 12.45 & 17.50 & 20.50 & 6.25 & 14.45 & 16.91 \\
\hline Sharp ratio & 0.75 & 0.50 & 0.94 & 1.13 & 0.37 & 0.89 & 1.00 \\
\hline C-2. Portfolio $\mathrm{p}$ & percentag & arn premia & the market port & ffolio & & & \\
\hline Monthly statisti & & & & & & & \\
\hline & $\begin{array}{l}\text { Small- } \\
\text { Low BM }\end{array}$ & $\begin{array}{l}\text { Small- } \\
\text { Neutral } \\
\end{array}$ & $\begin{array}{l}\text { Small- } \\
\text { High BM } \\
\end{array}$ & & $\begin{array}{l}\text { Big- } \\
\text { Low BM }\end{array}$ & $\begin{array}{l}\text { Big- } \\
\text { Neutral }\end{array}$ & $\begin{array}{l}\text { Big- } \\
\text { High BM }\end{array}$ \\
\hline Mean & 0.01 & 0.43 & 0.68 & & -0.51 & 0.17 & 0.38 \\
\hline Maximum & 11.06 & 6.73 & 6.67 & & 2.63 & 2.37 & 3.83 \\
\hline Minimum & -10.35 & -5.14 & -4.75 & & -4.43 & -1.77 & -4.54 \\
\hline SD & 4.44 & 2.64 & 2.54 & & 1.35 & 0.91 & 1.70 \\
\hline Skewness & 0.25 & 0.18 & 0.21 & & 0.04 & 0.48 & -0.61 \\
\hline Kurtosis & 0.31 & 0.09 & -0.31 & & 0.56 & 0.23 & 0.96 \\
\hline Annualized stat & tistics & & & & & & \\
\hline & $\begin{array}{l}\text { Small- } \\
\text { Low BM }\end{array}$ & $\begin{array}{l}\text { Small- } \\
\text { Neutral }\end{array}$ & $\begin{array}{l}\text { Small- } \\
\text { High BM }\end{array}$ & & $\begin{array}{l}\text { Big- } \\
\text { Low BM }\end{array}$ & $\begin{array}{l}\text { Big- } \\
\text { Neutral }\end{array}$ & $\begin{array}{l}\text { Big- } \\
\text { High BM }\end{array}$ \\
\hline Return premia & 0.07 & 5.12 & 8.11 & & -6.13 & 2.06 & 4.52 \\
\hline
\end{tabular}




\begin{tabular}{|c|c|c|c|c|c|c|c|}
\hline \multicolumn{8}{|c|}{ D-1. Portfolio percentage raw return } \\
\hline \multicolumn{8}{|c|}{ Monthly statistics } \\
\hline & Mkt & $\begin{array}{l}\text { Small- } \\
\text { Low BM }\end{array}$ & $\begin{array}{l}\text { Small- } \\
\text { Neutral }\end{array}$ & $\begin{array}{l}\text { Small- } \\
\text { High BM }\end{array}$ & $\begin{array}{l}\text { Big- } \\
\text { Low BM }\end{array}$ & $\begin{array}{l}\text { Big- } \\
\text { Neutral }\end{array}$ & $\begin{array}{l}\text { Big- } \\
\text { High BM }\end{array}$ \\
\hline Mean & -0.31 & -0.46 & -0.10 & 0.32 & -0.33 & -0.40 & -0.17 \\
\hline Maximum & 10.89 & 15.29 & 12.55 & 11.34 & 13.09 & 10.86 & 10.17 \\
\hline Minimum & -13.47 & -15.55 & -12.55 & -12.47 & -13.88 & -14.85 & -13.83 \\
\hline SD & 4.82 & 5.83 & 4.73 & 4.71 & 5.15 & 4.97 & 5.03 \\
\hline Skewness & -0.43 & -0.06 & -0.07 & 0.08 & -0.44 & -0.44 & -0.46 \\
\hline Kurtosis & 0.67 & 0.55 & 0.37 & 0.30 & 0.84 & 0.84 & 0.52 \\
\hline \multicolumn{8}{|c|}{ Annualized statistics } \\
\hline & Mkt & $\begin{array}{l}\text { Small- } \\
\text { Low BM }\end{array}$ & $\begin{array}{l}\text { Small- } \\
\text { Neutral }\end{array}$ & $\begin{array}{l}\text { Small- } \\
\text { High BM }\end{array}$ & $\begin{array}{l}\text { Big- } \\
\text { Low BM }\end{array}$ & $\begin{array}{l}\text { Big- } \\
\text { Neutral }\end{array}$ & $\begin{array}{l}\text { Big- } \\
\text { High BM }\end{array}$ \\
\hline Return & -3.72 & -5.48 & -1.26 & 3.86 & -3.98 & -4.82 & -2.09 \\
\hline SD & 16.68 & 20.20 & 16.38 & 16.33 & 17.85 & 17.20 & 17.42 \\
\hline Risk premia & -5.00 & -6.76 & -2.53 & 2.58 & -5.26 & -6.10 & -3.36 \\
\hline Sharp ratio & -0.30 & -0.33 & -0.15 & 0.16 & -0.29 & -0.35 & -0.19 \\
\hline \multicolumn{8}{|c|}{ D-2. Portfolio percentage return premia over the market portfolio } \\
\hline \multicolumn{8}{|c|}{ Monthly statistics } \\
\hline & $\begin{array}{l}\text { Small- } \\
\text { Low BM }\end{array}$ & $\begin{array}{l}\text { Small- } \\
\text { Neutral }\end{array}$ & \multicolumn{2}{|l|}{$\begin{array}{l}\text { Small- } \\
\text { High BM }\end{array}$} & $\begin{array}{l}\text { Big- } \\
\text { Low BM }\end{array}$ & $\begin{array}{l}\text { Big- } \\
\text { Neutral }\end{array}$ & $\begin{array}{l}\text { Big- } \\
\text { High BM }\end{array}$ \\
\hline Mean & -0.15 & 0.21 & \multicolumn{2}{|l|}{0.63} & -0.02 & -0.09 & 0.14 \\
\hline Maximum & 9.39 & 4.41 & \multicolumn{2}{|l|}{8.10} & 3.29 & 2.80 & 5.66 \\
\hline Minimum & -6.63 & -5.61 & \multicolumn{2}{|l|}{-5.96} & -3.53 & -2.69 & -3.27 \\
\hline SD & 3.02 & 2.10 & \multicolumn{2}{|l|}{2.65} & 1.43 & 0.96 & 1.69 \\
\hline Skewness & 0.48 & -0.23 & \multicolumn{2}{|l|}{-0.08} & 0.23 & -0.10 & 0.69 \\
\hline Kurtosis & 0.63 & -0.09 & 0.71 & & 0.25 & 0.91 & 1.19 \\
\hline \multicolumn{8}{|c|}{ Annualized statistics } \\
\hline & $\begin{array}{l}\text { Small- } \\
\text { Low BM }\end{array}$ & $\begin{array}{l}\text { Small- } \\
\text { Neutral }\end{array}$ & \multicolumn{2}{|l|}{$\begin{array}{l}\text { Small- } \\
\text { High BM }\end{array}$} & $\begin{array}{l}\text { Big- } \\
\text { Low BM }\end{array}$ & $\begin{array}{l}\text { Big- } \\
\text { Neutral }\end{array}$ & $\begin{array}{l}\text { Big- } \\
\text { High BM }\end{array}$ \\
\hline Return premia & -1.76 & 2.46 & 7.58 & & -0.26 & -1.10 & 1.63 \\
\hline Panel E. April & 2010 to $\mathrm{Ma}$ & 2015 & & & & & \\
\hline E-1. Portfolio 1 & ercentage $r$ & return & & & & & \\
\hline Monthly statist & & & & & & & \\
\hline & Mkt & $\begin{array}{l}\text { Small- } \\
\text { Low BM }\end{array}$ & $\begin{array}{l}\text { Small- } \\
\text { Neutral } \\
\end{array}$ & $\begin{array}{l}\text { Small- } \\
\text { High BM }\end{array}$ & $\begin{array}{l}\text { Big- } \\
\text { Low BM }\end{array}$ & $\begin{array}{l}\text { Big- } \\
\text { Neutral }\end{array}$ & $\begin{array}{l}\text { Big- } \\
\text { High BM }\end{array}$ \\
\hline Mean & 0.65 & 1.18 & 0.95 & 1.00 & 0.73 & 0.54 & 0.63 \\
\hline Maximum & 8.95 & 19.30 & 10.00 & 11.31 & 9.77 & 7.74 & 12.00 \\
\hline Minimum & -8.66 & -9.49 & -8.08 & -8.29 & -7.26 & -10.54 & -10.54 \\
\hline SD & 3.81 & 5.17 & 3.91 & 4.16 & 3.64 & 3.84 & 4.69 \\
\hline Skewness & -0.32 & 0.80 & 0.25 & 0.17 & -0.15 & -0.75 & -0.05 \\
\hline Kurtosis & 0.50 & 2.02 & 0.46 & 0.25 & 0.26 & 0.97 & 0.18 \\
\hline Annualized sta & tistics & & & & & & \\
\hline & Mkt & $\begin{array}{l}\text { Small- } \\
\text { Low BM }\end{array}$ & $\begin{array}{l}\text { Small- } \\
\text { Neutral } \\
\end{array}$ & $\begin{array}{l}\text { Small- } \\
\text { High BM }\end{array}$ & $\begin{array}{l}\text { Big- } \\
\text { Low BM }\end{array}$ & $\begin{array}{l}\text { Big- } \\
\text { Neutral }\end{array}$ & $\begin{array}{l}\text { Big- } \\
\text { High BM }\end{array}$ \\
\hline Return & 7.85 & 14.10 & 11.37 & 11.97 & 8.77 & 6.53 & 7.52 \\
\hline SD & 13.21 & 17.91 & 13.55 & 14.41 & 12.60 & 13.31 & 16.26 \\
\hline Risk premia & 7.81 & 14.06 & 11.34 & 11.94 & 8.73 & 6.49 & 7.48 \\
\hline Sharp ratio & 0.59 & 0.79 & 0.84 & 0.83 & 0.69 & 0.49 & 0.46 \\
\hline
\end{tabular}




\begin{tabular}{|c|c|c|c|c|c|c|c|}
\hline \multicolumn{8}{|c|}{ E-2. Portfolio percentage return premia over the market portfolio } \\
\hline \multicolumn{8}{|c|}{ Monthly statistics } \\
\hline & $\begin{array}{l}\text { Small- } \\
\text { Low BM }\end{array}$ & $\begin{array}{l}\text { Small- } \\
\text { Neutral }\end{array}$ & \multicolumn{2}{|l|}{$\begin{array}{l}\text { Small- } \\
\text { High BM }\end{array}$} & $\begin{array}{l}\text { Big- } \\
\text { Low BM }\end{array}$ & $\begin{array}{l}\text { Big- } \\
\text { Neutral }\end{array}$ & $\begin{array}{l}\text { Big- } \\
\text { High BM }\end{array}$ \\
\hline Mean & 0.52 & 0.29 & 0.34 & & 0.08 & -0.11 & -0.03 \\
\hline Maximum & 10.35 & 5.16 & \multicolumn{2}{|l|}{5.52} & 3.22 & 1.40 & 5.11 \\
\hline Minimum & -9.38 & -4.55 & \multicolumn{2}{|l|}{-4.08} & -2.51 & -1.88 & -3.36 \\
\hline SD & 3.07 & 1.96 & \multicolumn{2}{|l|}{2.20} & 1.05 & 0.72 & 1.54 \\
\hline Skewness & 0.08 & 0.04 & \multicolumn{2}{|l|}{0.24} & 0.51 & -0.37 & 0.74 \\
\hline Kurtosis & 2.32 & -0.04 & \multicolumn{2}{|l|}{-0.33} & 0.50 & 0.13 & 1.72 \\
\hline \multicolumn{8}{|c|}{ Annualized statistics } \\
\hline & $\begin{array}{l}\text { Small- } \\
\text { Low BM }\end{array}$ & $\begin{array}{l}\text { Small- } \\
\text { Neutral }\end{array}$ & \multicolumn{2}{|l|}{$\begin{array}{l}\text { Small- } \\
\text { High BM }\end{array}$} & $\begin{array}{l}\text { Big- } \\
\text { Low BM }\end{array}$ & $\begin{array}{l}\text { Big- } \\
\text { Neutral }\end{array}$ & $\begin{array}{l}\text { Big- } \\
\text { High BM }\end{array}$ \\
\hline Return premia & 6.25 & 3.53 & 4.13 & & 0.92 & -1.32 & -0.33 \\
\hline Panel F. April & 2015 to $\mathrm{Mar}$ & 2020 & & & & & \\
\hline F-1. Portfolio 1 & ercentage $r$ & return & & & & & \\
\hline Monthly statist & & & & & & & \\
\hline & Mkt & $\begin{array}{l}\text { Small- } \\
\text { Low BM } \\
\end{array}$ & $\begin{array}{l}\text { Small- } \\
\text { Neutral }\end{array}$ & $\begin{array}{l}\text { Small- } \\
\text { High BN }\end{array}$ & $\begin{array}{l}\text { Big- } \\
\text { Low BM }\end{array}$ & $\begin{array}{l}\text { Big- } \\
\text { Neutral }\end{array}$ & $\begin{array}{l}\text { Big- } \\
\text { High BM }\end{array}$ \\
\hline Mean & 0.32 & 0.58 & 0.61 & 0.51 & 0.55 & 0.25 & 0.04 \\
\hline Maximum & 9.26 & 11.99 & 7.60 & 8.42 & 10.63 & 9.11 & 9.58 \\
\hline Minimum & -9.97 & -17.32 & -13.64 & -13.35 & -11.71 & -8.95 & -11.65 \\
\hline SD & 3.72 & 5.29 & 4.08 & 3.98 & 4.07 & 3.59 & 4.55 \\
\hline Skewness & -0.60 & -1.14 & -1.05 & -0.90 & -0.95 & -0.36 & -0.31 \\
\hline Kurtosis & 0.82 & 2.35 & 2.04 & 1.67 & 1.64 & 0.44 & 0.10 \\
\hline Annualized sta & tistics & & & & & & \\
\hline & Mkt & $\begin{array}{l}\text { Small- } \\
\text { Low BM }\end{array}$ & $\begin{array}{l}\text { Small- } \\
\text { Neutral }\end{array}$ & $\begin{array}{l}\text { Small- } \\
\text { High BN }\end{array}$ & $\begin{array}{l}\text { Big- } \\
\text { Low BM }\end{array}$ & $\begin{array}{l}\text { Big- } \\
\text { Neutral }\end{array}$ & $\begin{array}{l}\text { Big- } \\
\text { High BM }\end{array}$ \\
\hline Return & 3.86 & 6.94 & 7.32 & 6.12 & 6.61 & 2.98 & 0.51 \\
\hline SD & 12.88 & 18.32 & 14.14 & 13.80 & 14.11 & 12.44 & 15.75 \\
\hline Risk premia & 2.81 & 5.88 & 6.26 & 5.07 & 5.55 & 1.92 & -0.55 \\
\hline Sharp ratio & 0.22 & 0.32 & 0.44 & 0.37 & 0.39 & 0.15 & -0.03 \\
\hline F-2. Portfolio 1 & ercentage $r$ & n premia or & he market portf & folio & & & \\
\hline Monthly statist & & & & & & & \\
\hline & $\begin{array}{l}\text { Small- } \\
\text { Low BM }\end{array}$ & $\begin{array}{l}\text { Small- } \\
\text { Neutral } \\
\end{array}$ & $\begin{array}{l}\text { Small- } \\
\text { High BM }\end{array}$ & & $\begin{array}{l}\text { Big- } \\
\text { Low BM }\end{array}$ & $\begin{array}{l}\text { Big- } \\
\text { Neutral }\end{array}$ & $\begin{array}{l}\text { Big- } \\
\text { High BM }\end{array}$ \\
\hline Mean & 0.26 & 0.29 & 0.19 & & 0.23 & -0.07 & -0.28 \\
\hline Maximum & 6.91 & 3.94 & 3.31 & & 5.75 & 1.32 & 4.78 \\
\hline Minimum & -7.94 & -3.67 & -4.08 & & -3.19 & -1.42 & -6.47 \\
\hline SD & 3.22 & 1.59 & 1.62 & & 1.66 & 0.64 & 2.09 \\
\hline Skewness & -0.43 & -0.18 & -0.20 & & 0.35 & 0.15 & 0.05 \\
\hline Kurtosis & 0.15 & -0.04 & -0.22 & & 1.18 & -0.25 & 0.72 \\
\hline Annualized sta & tistics & & & & & & \\
\hline & $\begin{array}{l}\text { Small- } \\
\text { Low BM }\end{array}$ & $\begin{array}{l}\text { Small- } \\
\text { Neutral } \\
\end{array}$ & $\begin{array}{l}\text { Small- } \\
\text { High BM }\end{array}$ & & $\begin{array}{l}\text { Big- } \\
\text { Low BM }\end{array}$ & $\begin{array}{l}\text { Big- } \\
\text { Neutral }\end{array}$ & $\begin{array}{l}\text { Big- } \\
\text { High BM }\end{array}$ \\
\hline Return premia & 3.08 & 3.46 & 2.26 & & 2.74 & -0.88 & -3.36 \\
\hline
\end{tabular}

Notes: The number of monthly observations analyzed in Panels A, B, C, D, E, and F are all 60. SD denotes standard deviation. 


\section{Conclusions}

The objective of this paper was to conduct an overview of stock portfolio returns and return premia in Japan by focusing on the size and BM portfolios. We computed many useful statistics and measures not only for our full sample period and the first half and second half sub-periods but also for different six five-year sub-periods. As we used data in US dollars and computed these statistics and measures, they should be highly beneficial not only for academic researchers but also for many international investors around the world.

As a result of our investigations, we conclude that in general, the value and/or size effects were continuously seen in the Japanese stock market; however, we stress that to obtain these effects to the maximum, market timing and investment horizon are crucial. This is because the economic and business circumstances are highly important, and these greatly affect the performance of size- and BM-sorted portfolios in Japan. Indeed, as our graphs showed, the value effect was stronger in our first half sub-period; while the size effect was much clearer in our second half sub-period. To sum up, when we start the investment and when we end the investment is highly crucial for investing the Japanese size- and BM-sorted portfolios.

Furthermore, we point out that the return characteristics in the Japanese stock market have changed; this can be seen that in Panels B-1 and C-1 of Table 1, the positive skewness values of the returns of both the market portfolio and the six size- and BM-sorted portfolios in our first half sub-period turned to all negative in our second half sub-period. We understand that this is a piece of evidence, indicating that the Japanese stock market have changed. Thus in the future, we should pay close attention to not only other international stock returns and return premia but also the tendency of the returns and return premia of stock portfolios in Japan.

Notably, although we explained only our main findings in Section 4; as exhibited in Tables 1 and 2, we also showed many other useful information on the Japanese stock portfolios for almost 30 years. We consider that all these statistics and measures not only for the full sample period but also for many other important sub-periods are highly beneficial for both academic researchers and industrial practitioners.

In the US, a recent study of Fama and French (2020) carefully attempted to examine whether the value premia still successively exist in more recent periods. As their research, the more detailed investigations may be required also for Japanese stock returns and return premia; and this line of research shall be one of our future works.

\section{Acknowledgements}

The author is particularly grateful to the repeated kind article invitation from this journal. The author also thanks James Young for his kind editorial assistance to my paper. Furthermore, the author thanks anonymous reviewers for their supportive comments on this paper, and I also appreciate a Grant-in-Aid for Scientific Research from the Japan Society for the Promotion of Science and the Chuo University Personal Research Grant for the generous financial assistance to this research. Finally, I deeply thank all the Editors of this journal for their kind attention and skillful editorship to my paper.

\section{References}

Chan, L. K. C., Hamao, Y., \& Lakonishok, J. (1991). Fundamentals and stock returns in Japan. Journal of Finance, 46, 1739-1764. https://doi.org/10.1111/j.1540-6261.1991.tb04642.x

Daniel, K., \& Titman, S. (1997). Evidence on the characteristics of cross sectional variation in stock returns. Journal of Finance, 52, 1-33. https://doi.org/10.1111/j.1540-6261.1997.tb03806.x

Daniel, K., Titman, S., \& Wei, K. C. J. (2001). Explaining the cross-section of stock returns in Japan: Factors or characteristics? Journal of Finance, 56, 743-766. https://doi.org/10.1111/0022-1082.00344

Fama, E. F., \& French, K. R. (1993). Common risk factors in the returns on stocks and bonds. Journal of Financial Economics, 33, 3-56. https://doi.org/10.1016/0304-405X(93)90023-5

Fama, E. F., \& French, K. R. (1995). Size and book-to-market factors in earnings and returns. Journal of Finance, 50, 131-155. https://doi.org/10.1111/j.1540-6261.1995.tb05169.x

Fama, E. F., \& French, K. R. (1998). Value versus growth: The international evidence. Journal of Finance, 53, 1975-1999. https://doi.org/10.1111/0022-1082.00080

Fama, E. F., \& French, K. R. (2012). Size, value, and momentum in international stock returns. Journal of Financial Economics, 105, 457-472. https:// doi.org/10.1016/j.jfineco.2012.05.011

Fama, E. F., \& French, K. R. (2017). International tests of a five-factor asset pricing model. Journal of Financial Economics, 123, 441-463. https://doi.org/10.1016/j.jfineco.2016.11.004

Fama, E. F., \& French, K. R. (2020). The value premium. Chicago Booth Paper, No. 20-01 (Fama-Miller Center for 
Research in Finance, The University of Chicago, Booth School of Business). https://doi.org/10.2139/ssrn.3525096

Tsuji, C. (2010). The limits of standard risk and macroeconomic factors in explaining the return premia: Evidence from the Tokyo Stock Exchange. International Business Research, 3, 80-96. https://doi.org/10.5539/ibr.v3n1p80

Tsuji, C. (2012). Positive return premia in Japan. Quantitative Finance, 12, $345-367$. https://doi.org/10.1080/14697688.2010.541485

\section{Copyrights}

Copyright for this article is retained by the author(s), with first publication rights granted to the journal.

This is an open-access article distributed under the terms and conditions of the Creative Commons Attribution license which permits unrestricted use, distribution, and reproduction in any medium, provided the original work is properly cited. 